\title{
Influência das variáveis antropométricas nas respostas cardiorrespiratórias de crianças durante o esforço
}

\author{
Influence of anthropometrics variables on the \\ cardiorespiratory responses of children during exercise
}

Fabiana Andrade Machado Benedito Sérgio Denadai ${ }^{2}$

1 Universidade Estadual de Maringá. Departamento de Educação Física. Maringá, PR. Brasil.

2 Universidade Estadual Paulista. Departamento de Educação Física. Rio Claro, SP. Brasil.

Recebido em 02/09/10 Revisado em 14/03/11 Aprovado em 08/04/11
Resumo - O objetivo deste estudo foi verificar a influência das diferentes dimensões corporais de meninos de 11 a 13 anos de idade, nas respostas cardiorrespiratórias, ao longo dos estágios de um teste incremental de esforço máximo em cicloergômetro. Vinte meninos realizaram um teste incremental máximo em cicloergômetro com carga inicial de $30 \mathrm{~W}$ e incrementos subsequentes de $30 \mathrm{~W}$ a cada três minutos. As variáveis respiratórias foram medidas respiração-a-respiracão através de um analisador metabólico de gases. A frequência cardíaca foi constantemente monitorada durante o teste. Os grupos foram divididos a posteriori em função da carga máxima atingida no teste incremental (90 ou 120 W) e em função da massa corporal (maior ou menor que $45 \mathrm{~kg}$ ). As seguintes variáveis foram mensuradas continuamente: frequência respiratória, volume corrente, ventilação, consumo de oxigênio absoluto e relativo, produção absoluta de gás carbônico, frequência cardíaca e equivalente ventilatório de oxigênio. Foi concluído que as variáveis antropométricas, especialmente estatura e massa corporal, mostram-se estreitamente relacionadas às respostas cardiorrespiratórias, apresentando-se como fatores determinantes e limitantes do desempenho, devendo ambas ser consideradas para a prescrição e prática de exercícios físicos desta população pediátrica.

Palavras-chave: Antropometria; Estatura; Exercício físico; Massa corporal.

Abstract - The objective of this study was to evaluate the influence of different body sizes on the cardiorespiratory responses of 10-13-year-old boys during the stages of a maximum-effort incremental test. Twenty boys underwent a continuous maximal incremental test on a cycle ergometer at an initial power output of $30 \mathrm{~W}$ and subsequent increases of $30 \mathrm{~W}$ at intervals of $3 \mathrm{~min}$. Respiratory variables were assessed with a metabolic gas analyzer on a breath-by-breath basis. Heart rate was monitored continuously during the test. The groups were divided a posteriori according to maximal work output in the incremental test $(90$ or $120 \mathrm{~W})$ and according to body weight (> 45 or $<45 \mathrm{~kg}$ ). The following variables were continuously measured: respiratory frequency, tidal volume, ventilation, absolute and relative oxygen uptake, carbon dioxide production, heart rate, and ventilatory equivalent for oxygen. The anthropometric variables, especially height and body weight, were closely associated with the cardiorespiratory responses. These variables are limiting and determinant factors of performance and need to be included in the prescription and practice of physical exercise for this pediatric population.

Key words: Anthropometry; Body weight; Height; Physical exercise. 


\section{INTRODUÇÃO}

A aptidão aeróbia pode ser definida como a capacidade de fornecer oxigênio aos músculos e de utilizá-lo para gerar energia durante os exercícios, dependendo, portanto, dos componentes pulmonares, cardiovasculares, hematológicos e dos mecanismos oxidativos do músculo em exercício. É determinada diretamente com o auxílio de um analisador metabólico de gases durante um teste ergoespirométrico em ergômetro apropriado, avaliando-se, simultaneamente, as capacidades do sistema cardiovascular e respiratório para realizar suas principais funções, tal como as trocas gasosas².

As mensurações das trocas gasosas são fundamentais para a compreensão dos mecanismos de limitação ao exercício físico, porque este requer uma resposta cardiopulmonar integrada para atender ao aumento das necessidades metabólicas dos músculos envolvidos em sua realização ${ }^{3}$.

$\mathrm{Na}$ puberdade, os processos de crescimento e desenvolvimento conferem um aumento nas dimensões corporais, com ênfase no desenvolvimento dos sistemas muscular e esquelético. Durante essa fase, ocorrem alterações hormonais que são responsáveis pelo aumento da massa muscular, especialmente em meninos, sendo coincidente com o pico de crescimento em estatura ${ }^{4}$. Esse aumento nas dimensões corporais implica também um aumento do tamanho pulmonar e do tamanho do coração alterando, portanto, os componentes ventilatórios e cardiovasculares das crianças 5 .

Crianças e adolescentes são submetidos a intensidades de esforço submáximas e máximas tanto em atividades de lazer, como em participações esportivas em nível competitivo, com exigências cada vez mais elevadas de desempenho. Observando as respostas fisiológicas e o comportamento das variáveis cardiorrespiratórias de crianças submetidas a esforços máximos, podemos compreender o desempenho peculiar desta clientela quando submetida a esforços físicos, de caráter recreacional ou competitivo e podemos também melhor prescrever intensidades adequadas de esforço, levando-se em consideração os fatores que podem ser limitantes e determinantes para o desempenho. Assim, faz-se necessário compreender e verificar as respostas fisiológicas que podem limitar ou contribuir com a prática de exercícios físicos e a prescrição de adequadas intensidades de esforço para crianças e adolescentes.

Desta forma, o objetivo deste estudo foi verificar a influência das diferentes dimensões corporais de meninos de 11 a 13 anos de idade, nas respostas cardiorrespiratórias, ao longo dos estágios de um teste incremental de esforço máximo realizado em cicloergômetro.

\section{PROCEDIMENTOS METODOLÓGICOS}

\section{Participantes}

Participaram deste estudo 20 meninos entre 11 e 13 anos de idade, aparentemente saudáveis e ativos. Nenhum dos participantes estava inserido em programas sistemáticos de treinamento. Todos os procedimentos adotados foram previamente aprovados pelo Comitê de Ética em Pesquisa local. A participação foi voluntária e isenta de qualquer bônus ou ônus aos sujeitos. Antes do início dos testes, os responsáveis tomaram conhecimento de todos os procedimentos a que as crianças seriam submetidas, assinando o termo de consentimento referente à participação nos mesmos.

\section{Teste incremental e variáveis cardiorrespiratórias}

Previamente à realização do teste incremental em cicloergômetro, os participantes realizaram uma visita no local da realização dos testes para uma sessão de adaptação ao cicloergômetro, na qual foram definidas as cargas inicial e de incremento para a realização dos testes. Nesta sessão, também foram feitas as mensurações da massa corporal e estatura.

O teste incremental contínuo foi realizado em um cicloergômetro de frenagem mecânica (Monark, São Paulo, Brasil), utilizando-se um assento anatômico para diminuir o desconforto dos participantes. A carga inicial foi de $30 \mathrm{~W}$ com incrementos subsequentes de $30 \mathrm{~W}$ a cada três minutos, mantendo-se uma cadência de $60 \mathrm{rpm}$. O cicloergômetro foi regulado e ajustado à estatura de cada participante, propiciando adequada angulação de joelhos e adequado posicionamento de tronco e membros inferiores e superiores. $O$ teste foi mantido até a exaustão voluntária, sendo os participantes encorajados verbalmente a se manterem em exercício pelo maior tempo possível, podendo ainda ser interrompido caso não fosse mantida a cadência estabelecida.

As variáveis respiratórias foram medidas utilizando-se um analisador de gases (Cosmed K4b², Roma, Itália), coletando dados respiração a respiração. Antes de cada teste, os sistemas de análise do $\mathrm{O}_{2}$ e $\mathrm{CO}_{2}$ e a turbina bi-direcional medidora de fluxo foram calibrados. Os dados obtidos respiração a respiração foram reduzidos a médias com 
intervalos de 15 segundos. A frequência cardíaca (FC) foi constantemente monitorada através de frequencímetro (Polar Vantage XL) durante todo o período do teste.

\section{Influência da dimensão corporal nas respostas cardiorrespiratórias durante o esforço}

Para verificar a influência das diferentes dimensões corporais nas respostas cardiorrespiratórias ao longo dos estágios do teste incremental, os participantes foram divididos em grupos, levando-se em consideração tanto a carga máxima atingida no teste incremental como a dimensão corporal dos avaliados. Esta divisão foi feita a posteriori, de posse da carga máxima de cada sujeito atingida no teste incremental. Apesar desta divisão em grupos tornar o número de sujeitos de cada grupo relativamente pequeno, esta divisão auxilia na análise e compreensão das respostas cardiorrespiratórias, levando-se em consideração as diferentes dimensões corporais apresentadas.

\section{Análise estatística}

Para a análise dos dados, foi utilizado o programa estatístico SPSS (Statistical Package for the Social Sciences) versão 17.0. Os dados são apresentados como média \pm desvio padrão (DP) para todas as variáveis mensuradas. A normalidade dos dados foi confirmada através do teste de Shapiro Wilk e a homocedasticidade foi verificada através do teste de Levene. A verificação de diferença estatística entre os grupos foi feita através do teste $t$ de Student bicaudal para dados não pareados na comparação entre dois grupos. Para a comparação entre mais que dois grupos foi utilizada a análise de variância simples (One way ANOVA), seguida pelo post hoc de Tukey quando necessário. O nível de significância estatística foi fixado em $\mathrm{p}<0,05$.

\section{RESULTADOS}

Os sujeitos foram divididos a posteriori de acordo com a massa corporal e a carga máxima atingida no teste incremental nos seguintes grupos: G1, composto de cinco meninos com massa corporal menor que $45 \mathrm{~kg}$ que atingiram a carga máxima de $90 \mathrm{~W}$ no teste incremental de esforço máximo; G2, composto de seis meninos com massa corporal menor que $45 \mathrm{~kg}$, mas que atingiram a carga máxima de $120 \mathrm{~W}$ no teste incremental; e G3, composto de seis meninos que atingiram a carga máxima de $120 \mathrm{~W}$ no teste incremental e que possuíam massa corporal superior a $45 \mathrm{~kg}$. Dentre os 20 sujeitos, um não completou o estágio de 90 W e dois completaram o estágio de $150 \mathrm{~W}$. Estes três sujeitos não foram incluídos nas análises e nos resultados apresentados por não pertencerem aos grupos acima definidos.

Na tabela 1, encontram-se os valores médios \pm DP das características antropométricas dos participantes (idade, massa corporal, estatura e índice de massa corporal - IMC) que compuseram a amostra total e subdivididos em grupos.

A massa corporal, estatura e IMC foram significantemente maiores no G3 em relação ao G1 e G2 $(\mathrm{p}<0,05)$. A estatura também foi estatisticamente maior no G2 em relação ao G1 ( $p<0,05)$.

A tabela 2 apresenta os valores médios das variáveis cardiorrespiratórias dos três grupos em função da intensidade de exercício no teste incremental (W).

\section{DISCUSSÃO}

O objetivo deste estudo foi verificar a influência das diferentes dimensões corporais de meninos de 11 a 13 anos de idade, nas respostas cardiorrespiratórias, ao longo dos estágios de um teste incremental de esforço máximo realizado em cicloergômetro.

Verifica-se, através da tabela 1, que apesar de G1, G2 e G3 apresentarem a mesma faixa etária, estes possuem valores crescentes tanto em massa corporal como em estatura e IMC. Os grupos foram divididos a posteriori, e esta divisão propiciou alcançar os objetivos deste experimento.

Tabela 1. Valores médios \pm DP das características dos participantes: idade (anos), massa corporal (kg), estatura (m) e índice de massa corporal - IMC $\left(\mathrm{kg} / \mathrm{m}^{2}\right)$ que compuseram a amostra total e subdivididos em grupos.

\begin{tabular}{lcccc}
\hline GRUPOS & IDADE $($ anos $)$ & MASSA CORPORAL $(\mathrm{kg})$ & ESTATURA $(\mathrm{m})$ & $\mathrm{IMC}\left(\mathrm{kg} / \mathrm{m}^{2}\right)$ \\
\hline Amostra total $(\mathrm{n}=17)$ & $11,75 \pm 0,28$ & $40,94 \pm 10,21$ & $1,47 \pm 0,07$ & $18,63 \pm 3,44$ \\
G1 $(\mathrm{n}=5)$ & $11,72 \pm 0,35$ & $33,04 \pm 4,72$ & $1,41 \pm 0,05$ & $16,60 \pm 1,28$ \\
G2 $(\mathrm{n}=6)$ & $11,87 \pm 0,25$ & $36,07 \pm 4,04$ & $1,47 \pm 0,02^{*}$ & $16,72 \pm 1,73$ \\
G3 $(\mathrm{n}=6)$ & $11,68 \pm 0,23$ & $52,40 \pm 7,15^{* *}$ & $1,54 \pm 0,05^{* *}$ & $22,23 \pm 3,06^{* *}$ \\
\hline
\end{tabular}

* $p<0,05$ em relação ao G1; ** p<0,05 em relação ao G1 e G2. 
Tabela 2. Valores médios das variáveis cardiorrespiratórias dos três grupos em função da intensidade de exercício no teste incremental (W).

\begin{tabular}{|c|c|c|c|c|c|c|c|c|c|}
\hline \multicolumn{5}{|c|}{ Frequência respiratória ciclos/min) } & & \multicolumn{4}{|c|}{ Volume corrente (I) } \\
\hline & $30 \mathrm{~W}$ & $60 \mathrm{~W}$ & $90 \mathrm{~W}$ & $120 \mathrm{~W}$ & & $30 \mathrm{~W}$ & $60 \mathrm{~W}$ & $90 \mathrm{~W}$ & $120 \mathrm{~W}$ \\
\hline G1 & 32,3 & 40,0 & 51,5 & - & G1 & 0,62 & 0,86 & 1,00 & - \\
\hline G2 & 31,6 & 37,3 & 45,7 & 56,1 & G2 & 0,71 & 0,96 & 1,10 & 1,29 \\
\hline G3 & 30,7 & 35,2 & 41,8 & 49,2 & G3 & 0,77 & 1,02 & 1,19 & 1,40 \\
\hline \multicolumn{5}{|c|}{ Ventilação (l/min) } & & \multicolumn{4}{|c|}{ Consumo de oxigênio relativo $\left(\mathrm{ml} / \mathrm{kg}^{0,80} / \mathrm{min}\right)$} \\
\hline & $30 \mathrm{~W}$ & $60 \mathrm{~W}$ & $90 \mathrm{~W}$ & $120 \mathrm{~W}$ & & $30 \mathrm{~W}$ & $60 \mathrm{~W}$ & $90 \mathrm{~W}$ & $120 \mathrm{~W}$ \\
\hline G1 & 20,0 & 33,9 & 51,0 & - & G1 & 40,8 & 68,9 & 82,2 & - \\
\hline G2 & 21,7 & 35,3 & 48,6 & 72,3 & G2 & 42,8 & 69,4 & 79,8 & 91,5 \\
\hline G3 & 23,6 & 35,6 & 49,6 & 68,6 & G3 & 37.8 & $56,1^{* * *}$ & $67.8^{*}$ & 81,9 \\
\hline \multicolumn{5}{|c|}{ Frequência cardíaca (bpm) } & & \multicolumn{4}{|c|}{ Produção de gás carbônico $(\mathrm{ml} / \mathrm{min})$} \\
\hline & $30 \mathrm{~W}$ & $60 \mathrm{~W}$ & $90 \mathrm{~W}$ & $120 \mathrm{~W}$ & & $30 \mathrm{~W}$ & $60 \mathrm{~W}$ & $90 \mathrm{~W}$ & $120 \mathrm{~W}$ \\
\hline G1 & 121 & 151 & 177 & - & G1 & 520 & 970 & 1345 & - \\
\hline G2 & 117 & 142 & 165 & 184 & G2 & 612 & 1073 & 1361 & 1780 \\
\hline G3 & 120 & 143 & 164 & 181 & G3 & $723^{*}$ & 1175 & 1534 & $2004^{* *}$ \\
\hline \multicolumn{5}{|c|}{ Consumo de oxigênio absoluto (ml/min) } & & \multicolumn{4}{|c|}{ Equivalente ventilatório de oxigênio } \\
\hline & $30 \mathrm{~W}$ & $60 \mathrm{~W}$ & $90 \mathrm{~W}$ & $120 \mathrm{~W}$ & & $30 \mathrm{~W}$ & $60 \mathrm{~W}$ & $90 \mathrm{~W}$ & $120 \mathrm{~W}$ \\
\hline G1 & 663 & 1121 & 1344 & & G1 & 26,6 & 27,8 & 35,5 & \\
\hline G2 & 752 & 1217 & 1392 & 1599 & G2 & 26,0 & 26,9 & 32,9 & 42,9 \\
\hline G3 & $898^{*}$ & 1331 & $1604^{*}$ & $1932^{* *}$ & G3 & 24,0 & 25,0 & $29,1^{*}$ & $33,8^{* *}$ \\
\hline
\end{tabular}

* $\mathrm{p}<0,05$ em relação ao G1; ** $\mathrm{p}<0,05$ em relação ao G2; ${ }^{* * *} \mathrm{p}<0,05$ em relação ao G1 e G2.

Conforme a tabela 2, o volume corrente (VC) foi crescente do G1 para o G3 ao longo de todas as intensidades de exercício. O maior valor do VC para o G3 está relacionado com a maior dimensão corporal deste grupo. A maior parte do desenvolvimento dos componentes respiratórios ocorre paralelamente aos aumentos do tamanho pulmonar $^{5}$, sendo que este se correlaciona estreitamente tanto com a estatura como com a estatura ao cubo de acordo com Lyons e Tanner ${ }^{6}$. Neste estudo, as correlações do VC com a estatura e com a massa corporal foram similares, porém a correlação com a estatura foi levemente maior. $\mathrm{Na}$ intensidade de 60 $\mathrm{W}$, por exemplo, a correlação do VC com a estatura de 0,64 e a correlação com a massa corporal foi 0,59. O VC aumentou nos três grupos linearmente com o aumento da intensidade de esforço. A taxa média desse aumento foi de $7 \mathrm{ml} / \mathrm{W}$.

Em relação à ventilação (VE), verifica-se, na tabela 2, que não houve diferença estatística entre os grupos em cada intensidade de exercício. Esta demonstrou ser influenciada pela intensidade de exercício, ocorrendo um aumento linear $\left(\mathrm{R}^{2}=0,99\right)$ a uma taxa de $0,535 \mathrm{l} / \mathrm{W} / \mathrm{min}$ com o aumento da intensidade de exercício. A VE $\mathrm{VE}_{\text {máx }}(1 / \mathrm{kg} / \mathrm{min})$ foi de 1,54 (G1); 2,00 (G2) e 1,31(G3), aparentando decrescer com o aumento da massa corporal para uma mesma intensidade máxima de exercício. Este resultado está de acordo com Mercier et al. ${ }^{7}$ e Armostrong ${ }^{8}$ que também verificaram o declínio da
$\mathrm{VE}_{\text {máx }}(1 / \mathrm{kg} / \mathrm{min})$ com o aumento da massa corporal. Em relação à frequencia respiratória (FR), esta, ao contrário do VC, decresceu do G1 para o G3. Como a VE foi praticamente a mesma para os três grupos, em cada intensidade de exercício e esta é definida como o produto da FR pelo VC, então a FR se comportou de forma inversamente proporcional a VC. Desta forma, a FR se relacionou tanto com a estatura quanto com a massa corporal indiretamente por meio do $\mathrm{VC}$, de forma inversamente proporcional para cada intensidade de exercício. A FR pico do G1 foi semelhante a do G3 apesar dos meninos do G1 terem atingido a carga máxima de $90 \mathrm{~W}$ enquanto os meninos do G3 atingiram a carga máxima de $120 \mathrm{~W}$.

Os valores absolutos de VC, no exercício máximo, aumentam com o crescimento de acordo com as maiores dimensões do pulmão. A razão da FR com o VC no pico do exercício, declina para uma maior dimensão corporal. Encontramos os seguintes valores para a razão FR/VC na intensidade máxima de exercício: 51,5 (G1); 43,5 (G2) e 35,1 ciclos/1/min (G3). Desta forma, a razão FR/VC no pico do exercício declinou aproximadamente $32 \%$ do G1 para o G3. Assim, da mesma forma que Rowland e Cunningham ${ }^{9}$, obtivemos valores decrescentes entre a FR/VC com o aumento da estatura e massa corporal.

De acordo com a tabela 2, a FC aumentou linearmente com o exercício progressivo para os 
três grupos, obtendo-se um coeficiente de correlação entre a $\mathrm{FC}$ e a intensidade de exercício maior que 0,99 para os três grupos. De acordo com Rowland ${ }^{5}$, a FC em uma dada intensidade de trabalho declina conforme as crianças crescem. Isso reflete o aumento do tamanho do coração e do volume sistólico (VS). Uma vez que a demanda de oxigênio e o débito cardíaco (DC), necessários para uma carga de trabalho especifica, permanece a mesma, independente do tamanho corporal, uma criança maior satisfaz esses requisitos por meio de uma maior dependência do VS e menor da FC do que as crianças menores 5 . Entretanto, de acordo com a tabela 2, apesar da FC ter sido menor para o G3 em relação ao G1, para uma mesma intensidade de exercício, esta diferença não foi estatisticamente significante ( $p>0,05)$. Na comparação do G2 e G3 que apresentaram uma massa corporal média de $36,07 \pm 4,04$ e $52,40 \pm 7,15$, respectivamente, não houve praticamente nenhuma diferença na FC em todas as intensidades de exercício. Deste modo, diferentemente da afirmação de Rowland ${ }^{5}$, os nossos resultados não demonstraram, pelo menos em exercícios no cicloergômetro, que a FC dependa da massa corporal para uma mesma intensidade submáxima de exercício.

$\mathrm{O}$ consumo de oxigênio $\left(\mathrm{VO}_{2}\right)$ relativo foi relacionado à massa corporal ao expoente 0,80 $\left(\mathrm{ml} / \mathrm{kg}^{0,80} / \mathrm{min}\right)$. Evitou-se expressar o $\mathrm{VO}_{2}$ dividido simplesmente pela massa corporal ( $\mathrm{ml} / \mathrm{kg} / \mathrm{min}$ ) por questões de alometria. Sabe-se que a simples divisão do $\mathrm{VO}_{2}$ pela massa corporal não elimina o efeito do tamanho corporal sobre o $\mathrm{VO}_{2}$. Assim, esta simples divisão não é apropriada para ajustar o tamanho corporal para comparações de valores de $\mathrm{VO}_{2}$ interou intraindividuais. Segundo Rowland ${ }^{5}$, quando o $\mathrm{VO}_{2}$ max é relacionado à massa corporal por análise alométrica, a maioria dos expoentes encontrados na literatura varia de 0,70 a 0,90 . Welsman et al..$^{10}$ encontraram o expoente 0,80 e desta forma adotamos neste estudo este mesmo expoente como forma de eliminar o efeito do tamanho corporal no $\mathrm{VO}_{2}$.

Verifica-se que o $\mathrm{VO}_{2}$ relativo $\left(\mathrm{ml} / \mathrm{kg}^{0,80} / \mathrm{min}\right)$ se mostrou decrescente entre G1 e G3 para as mesmas intensidades de exercício (tabela 2). Como os testes foram realizados em cicloergômetro, não foi necessário a sustentação do corpo como na corrida, e assim, estas diferenças se devem, principalmente, à economia de movimento para a realização do exercício. Sabe-se que a potência muscular e a eficiência mecânica melhoram com o crescimento e desenvolvimento ${ }^{5}$. Verifica-se que $\mathrm{G} 1$ e G3 apresentaram um $\mathrm{VO}_{2}$ pico semelhante, apresentando, portanto, uma potência aeróbia semelhante. Entretanto, o G3 completou um estágio a mais que o G1 no teste incremental. Quando comparamos o $\mathrm{VO}_{2}$ relativo $\left(\mathrm{ml} / \mathrm{kg}^{\mathrm{0}, 80}\right)$ $\mathrm{min}$ ) na intensidade de $60 \mathrm{~W}$, para estes dois grupos com potência aeróbia semelhante, verificamos que esta intensidade é muito mais baixa para o G3 (68\% $\mathrm{VO}_{2}$ pico) do que para o $\mathrm{G1}\left(84 \% \mathrm{VO}_{2}\right.$ pico). Assim, caso a intensidade de $60 \mathrm{~W}$ seja prescrita para ambos os grupos sem levar em consideração o tamanho corporal, se esta intensidade for adequada para o G1 ela será baixa para o G3 devido, principalmente, à eficiência mecânica (economia de movimento). Desta forma, o tamanho corporal deve ser levado em conta para a prescrição da adequada intensidade de exercício para a população pediátrica aqui estudada, visto que a economia de movimento melhora com o crescimento e o desenvolvimento.

Tanto o $\mathrm{VO}_{2}$ absoluto quanto a produção de gás carbônico $\left(\mathrm{VCO}_{2}\right)$ absoluta aumentaram entre G1 e G3 (tabela 2). Estas diferenças, que ficam mais acentuadas comparando-se o G3 com o G1, se devem principalmente à diferença na massa corporal. O equivalente ventilatório representa quantos litros de ar precisam ser ventilados para que um litro de oxigênio ou gás carbônico sejam, respectivamente, consumidos ou produzidos. Como verificamos que a ventilação depende, principalmente, da intensidade de esforço e variou pouco entre os grupos, o equivalente de $\mathrm{O}_{2}$ foi inversamente proporcional ao $\mathrm{VO}_{2}$ absoluto para determinada intensidade de esforço. Assim, como o $\mathrm{G} 3$ apresentou um maior $\mathrm{VO}_{2}$ absoluto, o mesmo também demonstrou um menor equivalente ventilatório de oxigênio, necessitando de uma menor ventilação em relação aos outros grupos para o consumo de um litro de oxigênio.

\section{CONCLUSÕES}

Desta forma, concluímos que as variáveis antropométricas, especialmente estatura e massa corporal, mostram-se estreitamente relacionadas às respostas cardiorrespiratórias, apresentando-se como fatores determinantes e limitantes do desempenho de meninos entre 11 e 13 anos de idade. Adicionalmente, verificou-se que a carga de exercício prescrita para meninos com aptidão aeróbia semelhantes, mas com tamanhos corporais diferentes, deve ser proporcional ao tamanho corporal de forma que se leve em consideração a melhora na economia de movimento que acompanha os processos de crescimento e desenvolvimento. 


\section{REFERÊNCIAS BIBLIOGRÁFICAS}

1. Armstrong N. Aerobic fitness of children and adolescents. J Pediatr (Rio J) 2006;82:406-8.

2. Armstrong N, Welsman JR. Assesment and interpretation of aerobic fitness in children and adolescents. Exerc Sport Sci Rev 1994;22:435-76.

3. Rodrigues AN, Perez AJ, Carletti L, Bissoli NS, Abreu GR. Maximum oxygen uptake in adolescents as measured by cardiopulmonary exercise testing: a classification proposal. J Pediatr (Rio J) 2006;82:426-30.

4. Tanner JM, Hughes PC, Whitehouse RH. Radiographically determined widths of bone muscle and fat in the upper arm and calf from age 3-18 years. Ann Hum Biol 1981;8:495-517.

5. Rowland TW. Fisiologia do exercício na criança. $2^{\mathrm{a}}$. ed. Barueri: Manole, 2008.

6. Lyons HA, Tanner RW. Total lung volume and its subdivisions in children: normal standards. J Appl Physiol 1962;17:601-4.

7. Mercier J, Varray A, Ramonatxo M, Mercier B, Préfaut C. Influence of anthropometric characteristics on changes in maximal exercise ventilation and breathing pattern during growth in boys. Eur J Appl Physiol Occup Physiol 1991;63:235-41.
8. Armstrong N, Kirby BJ, McManus AM, Welsman JR. Prepubescents' ventilatory responses to exercise with reference to sex and body size. Chest 1997;112:1554-60.

9. Rowland TW, Cunningham LN. Development of ventilatory responses to exercise in normal white children. A longitudinal study. Chest 1997;11:327-32.

10. Welsman JR, Armstrong N, Nevill AM, Winter EM, Kirby BJ. Scaling peak VO2 for differences in body size. Med Sci Sports Exerc 1996;28:259-65.
Endereço para correspondência
Fabiana Andrade Machado
Universidade Estadual de Maringá.
Departamento de Educação Física
Av. Colombo, 5790 - CEP: 87.020-900
Maringá, PR. Brasil
E-mail: famachado@uem.br; famachado_uem@hotmail.com 